\title{
Protein Tyrosine Phosphatase, Receptor Type B (PTPRB) Inhibits Brown Adipocyte Differentiation through Regulation of VEGFR2 Phosphorylation
}

\author{
Ji Soo Kim ${ }^{1,2 \dagger}$, Won Kon Kim ${ }^{1,2 \dagger}$, Kyoung-Jin $\mathrm{Oh}^{1,2}$, Eun-Woo Lee ${ }^{1}$, Baek Soo Han ${ }^{1,2}$, Sang Chul Lee ${ }^{1,2 *}$, and \\ Kwang-Hee Bae ${ }^{1,2 *}$ \\ ${ }^{1}$ Metabolic Regulation Research Center, Division of BioMedical Sciences, KRIBB, Daejeon 34141, Republic of Korea \\ ${ }^{2}$ Department of Functional Genomics, University of Science and Technology (UST) of Korea, Daejeon 34141, Republic of Korea
}

Received: October 18, 2018

Revised: January 25, 2019

Accepted: February 11, 2019

First published online

February 20, 2019

${ }^{*}$ Corresponding authors

S.C.L.

Phone: +82-42-860-4142;

Fax: +82-42-860-4593;

E-mail: lesach@kribb.re.kr

K.-H.B.

Phone: +82-42-860-4268;

Fax: +82-42-860-4593;

E-mail: khbae@kribb.re.kr

${ }^{\dagger}$ These authors contributed

equally to this work.

pISSN 1017-7825, eISSN 1738-8872

Copyright(C) 2019 by

The Korean Society for Microbiology and Biotechnology

\begin{abstract}
Brown adipocytes have an important role in the regulation of energy balance through uncoupling protein-1 (UCP-1)-mediated nonshivering thermogenesis. Although brown adipocytes have been highlighted as a new therapeutic target for the treatment of metabolic diseases, such as obesity and type II diabetes in adult humans, the molecular mechanism underlying brown adipogenesis is not fully understood. We recently found that protein tyrosine phosphatase receptor type B (PTPRB) expression dramatically decreased during brown adipogenic differentiation. In this study, we investigated the functional roles of PTPRB and its regulatory mechanism during brown adipocyte differentiation. Ectopic expression of PTPRB led to a reduced brown adipocyte differentiation by suppressing the tyrosine phosphorylation of VEGFR2, whereas a catalytic inactive PTPRB mutant showed no effects on differentiation and phosphorylation. Consistently, the expression of brown adipocyte-related genes, such as UCP-1, PGC-1 $\alpha$, PRDM16, PPAR- $\gamma$, and CIDEA, were significantly inhibited by PTPRB overexpression. Overall, these results suggest that PTPRB functions as a negative regulator of brown adipocyte differentiation through its phosphatase activity-dependent mechanism and may be used as a target protein for the regulation of obesity and type II diabetes.
\end{abstract}

Keywords: Brown adipogenesis, obesity, PTPRB, VEGFR2

\section{Introduction}

Obesity is a worldwide challenge and is closely associated with metabolic diseases, such as type II diabetes, dyslipidemia, fatty liver and cardiovascular diseases [1, 2]. Obesity is caused by an energy imbalance between the calories consumed and calories expended. Therefore, the reduction of energy intake and/or the increase of energy expenditure are prominent goals to counteract obesity.

Adipose tissue is a major metabolic organ composed of white adipose tissue (WAT) and brown adipose tissue (BAT). The two tissues are both involved in energy balance. WAT stores excess energy in the form of triglycerides (TGs), whereas BAT oxidizes fuels and dissipates energy in the form of heat by uncoupling mitochondrial respiration from ATP production through the expression and activation of the brown fat-specific uncoupling protein-1 (UCP-1) [2, 3-6]. Thus, BAT has a pivotal role in nonshivering thermogenesis to protect against energy overload. In addition, understanding the molecular mechanism of brown adipocyte differentiation may provide new ways to treat obesity and obesity-related metabolic diseases. However, the regulation of brown adipogenesis remains poorly understood until now.

Tyrosine phosphorylation is an important post-translational modification that regulates signaling events in multiple cells by opposing the activities of protein tyrosine kinases (PTKs) and protein tyrosine phosphatases (PTPs). PTKs 
catalyze tyrosine phosphorylation, and PTPs are responsible for tyrosine dephosphorylation [1, 7-9]. In particular, PTPs have a more active role in many key cellular processes including differentiation. Previously, we revealed that several PTPs have a pivotal role in the regulation of adipocyte differentiation and osteogenic differentiation [10-15]. Recently, we investigated the expression of all PTPs during adipogenic differentiation of primary brown preadipocytes. Among them, protein tyrosine phosphatase receptor type B (PTPRB) expression was dramatically decreased [16]. PTPRB is transmembrane receptor type PTP, which is known as a vascular endothelial protein tyrosine phosphatase (VE-PTP). PTPRB is well known to be involved in the maintenance and remodeling of blood vessels and in angiogenesis [17, 18], and it also dephosphorylates vascular endothelial growth factor receptor 2 (VEGFR2) [19]. Although vascular endothelial growth factor (VEGF) is involved in brown adipocyte differentiation [20], there are no direct reports on the relationship between brown adipogenesis and PTPRB. In this study, we focused on the functional roles of PTPRB during the differentiation of immortalized preadipocytes derived from BAT. We show that PTPRB inhibits brown adipocyte differentiation through its phosphatase activity-dependent mechanism.

\section{Materials and Methods}

\section{Cell Culture and Differentiation of Immortalized Brown Preadipocytes}

The immortalized brown preadipocyte cell line was kindly provided by Dr. Shingo Kajimura (UCSF). Cells were grown in Dulbecco's modified Eagle's medium (DMEM) containing 1\% antibiotic/antimycotic solution and $10 \%$ fetal bovine serum (FBS) at $37^{\circ} \mathrm{C}$ in a humidified atmosphere with $5 \% \mathrm{CO}_{2}$. The immortalized brown preadipocytes were induced to differentiate into mature brown adipocytes by a previously described protocol [21, 22]. Lipid droplets in differentiating or mature brown adipocytes were stained using the Oil-Red-O method, as described previously [22].

\section{Overexpression of PTPRB in Brown Preadipocytes}

To establish that PTPRB was stably expressed, a retrovirusmediated infection system was used. The Flag-tagged PTPRB was inserted into the multi-cloning site of the pRetroX-IRES-ZsGreen1 vector (Clontech). The catalytically inactive mutant of PTPRB, in which Cys-1904 was replaced by Ser, was constructed by sitedirected mutagenesis using a QuikChange Mutagenesis Kit (Stratagene) according to the manufacturer's instructions. For viral production, GP2-293 cell lines were transfected using Lipofectamine 2000 (Life Technologies-Invitrogen). The details of the transfection and transduction methods were described previously $[12,23]$. Infected cells were selected using a fluorescence-activated cell sorting system (FACSAria cell sorter, BD Biosciences) and further maintained in a growth medium.

\section{Quantitative PCR}

Total RNA was extracted from cultured cells using Trizol according to the manufacturer's instructions, and first-strand cDNA was synthesized from total RNA using Reverse Transcriptase M-MLV (Promega) according to the manufacturer's instructions. The targeted fragment of cDNA for the brown adipocyte-related genes was amplified by quantitative real-time PCR using each primer [15]. The gene expression level was normalized to that of the TBP and L32 genes in the same sample.

\section{Immunoblot Analysis}

Cells were washed three times with ice-cold PBS containing $1 \mathrm{mM}$ sodium orthovanadate and harvested in RIPA lysis buffer containing a protease-inhibitor and phosphatase-inhibitor cocktail (Roche). Protein concentrations were measured with the Bradford assay (Bio-Rad). SDS-PAGE and western blot analysis were performed using a standard procedure [24, 25]. Antibodies against VEGFR2 and phospho-VEGFR2 (Tyr1175) were obtained from Cell Signaling. The anti-Flag was from Sigma, and the HSP90 antibody was purchased from Santa Cruz. The secondary antibodies were obtained from Abcam, and the membranes were visualized using an enhanced chemiluminescence system (Amersham). CP547365, a pharmacological inhibitor of VEGFR2 tyrosine phosphorylation, was acquired from Sigma.

\section{Mitochondrial Staining and Quantification of Mitochondrial DNA}

For quantification of mitochondrial DNA (mtDNA), total genomic DNA was isolated using the Exgene Tissue SV DNA Mini Kit (Geneall, Korea) from brown adipocytes. Then, the mtDNA was amplified using primers for NADH dehydrogenase 1 (ND1) and normalized to the genomic DNA by primers amplifying the TATA box binding protein (TBP) from the genomic DNA as previously described [22]. To confirm the mitochondrial amount and distribution, cells were stained with MitoTracker using a previously described method [22]. Stained cells were detected by confocal microscopy, and fluorescence was measured at a wavelength of $450-550 \mathrm{~nm}$.

\section{Results and Discussion}

The immortalized brown preadipocyte cell line was kindly provided by Dr. Shingo Kajimura (UCSF, USA). Previously, we have successfully induced the differentiation of immortalized brown preadipocytes into mature brown adipocytes $[15,26]$. Consistently, the expression levels of brown adipogenic markers, such as UCP-1, PGC-1 $\alpha$, PRMD16 and PPAR- $\gamma$, were significantly increased during brown adipocyte differentiation (data not shown). In 
A

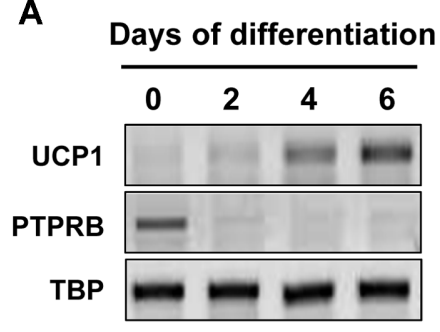

B.

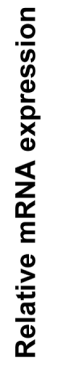

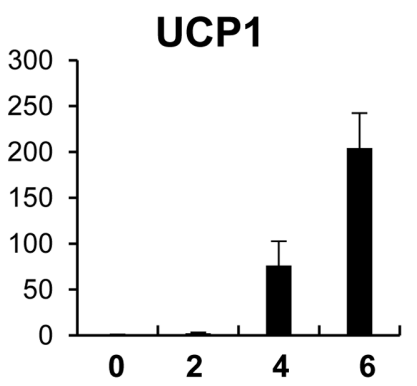

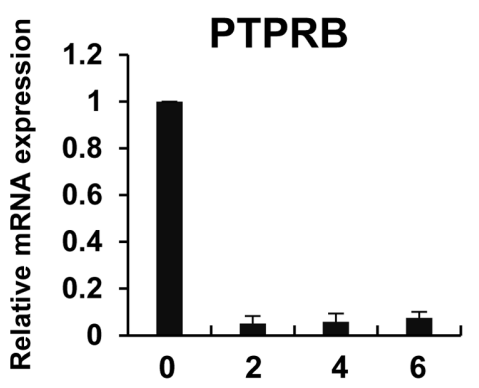

\section{Days of differentiation}

Fig. 1. The analysis of the PTPRB expression during differentiation of the immortalized brown preadipocytes.

Quantification of PTPRB mRNA expression during brown adipocyte differentiation by RT-PCR (A) and Real-time PCR (B). Total RNA was extracted on the indicated days of differentiation. TBP was used as a loading control.

contrast, PTPRB expression dramatically decreased during the differentiation of the immortalized brown preadipocytes (Fig. 1). Recently, Xue et al. [27] reported that the expression of UCP-1 and PTPRB had a negative correlation indicating that PTPRB may have a potentially negative role during brown adipogenesis.
To identify the functional roles of PTPRB in brown adipocyte differentiation, immortalized brown preadipocytes were infected with FLAG-tagged PTPRB using a retrovirus system (PTPRB IRES-GFP). Additionally, to determine the importance of the phosphatase activity of PTPRB, a virus with a catalytic inactive mutant of PTPRB was also
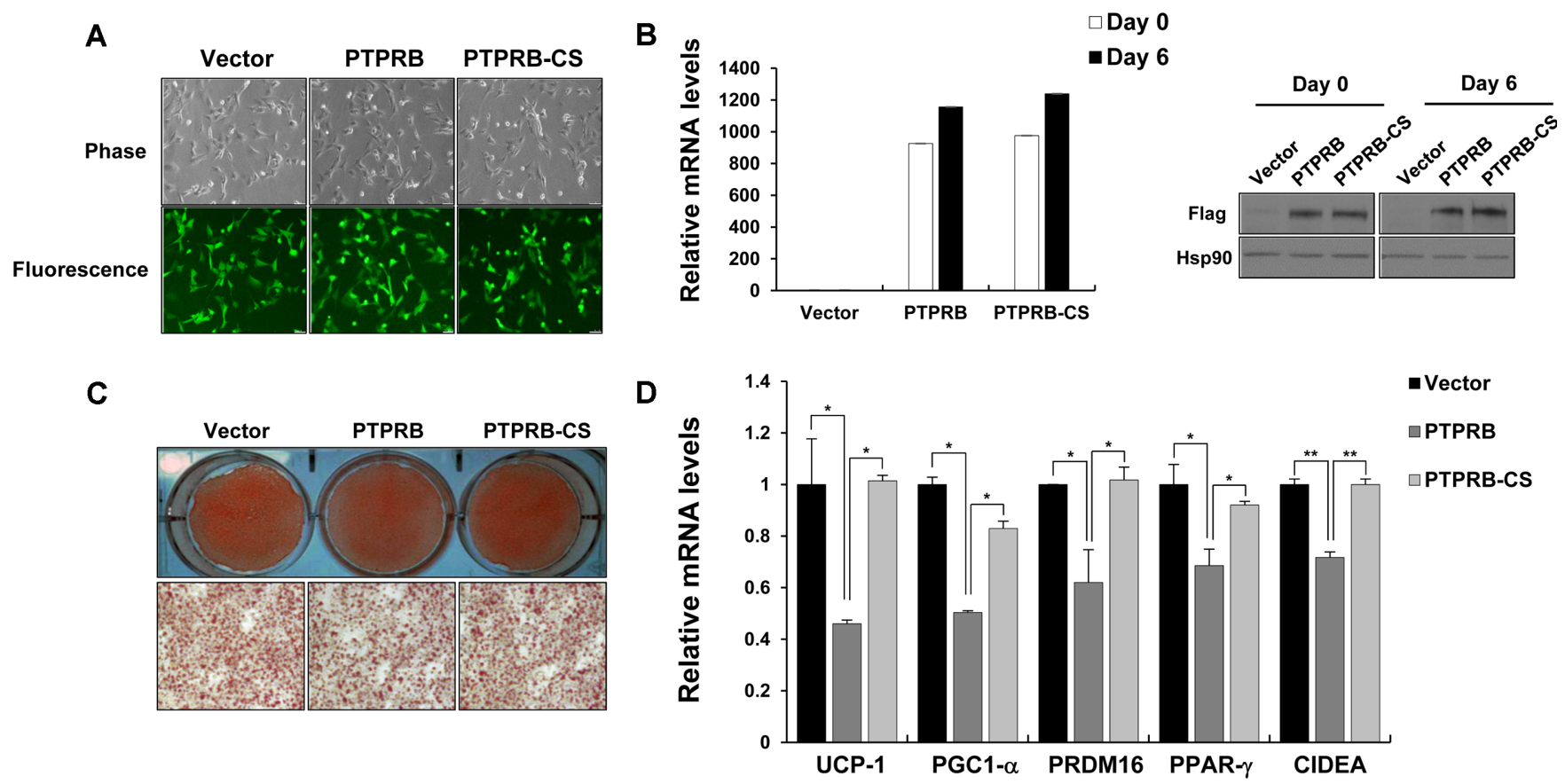

Fig. 2. The overexpression of PTPRB suppresses brown adipocyte differentiation of the immortalized brown preadipocytes. Immortalized brown preadipocytes were stably infected with retroviruses (pRetroX-IRES-ZsGreen1) expressing Control Vector, FLAG-tagged PTPRB or PTPRB-CS (catalytically inactive; catalytic Cys 1907 was replaced with Ser). Infected cells were selected by FACS sorting. (A) GFP expression was monitored directly using fluorescence microscopy. (B) The expression level of PTPRB was confirmed by Real-time PCR and western blot analysis. (C) The cells expressing wild-type or mutant PTPRB were induced to differentiate into brown adipocytes through the brown adipogenic program for 6 days. Then, cells were stained with Oil-Red-O to visualize the lipid droplets. (D) The mRNA expression levels of UCP1, PGC-1 $\alpha$, PRDM16, PPAR- $\gamma$, and CIDEA were analyzed by Real-time PCR. The data represent the means $\pm \operatorname{SD}\left(n=3,{ }^{*} p<0.05\right)$. 
constructed (PTPRB-CS; catalytic Cys1904 was replaced with Ser). The infected cells were isolated using a FACSAria sorter (FACSAria, BD Biosciences) and further grown. Most of the enriched cells were green fluorescent protein (GFP) positive under a fluorescence microscope (Fig. 2A). Ectopic expression of the wild-type and mutant PTPRB was verified by western blot and real-time PCR. Expression of the PTPRB and PTPRB-CS was continuously detected in mature brown adipocytes (Fig. 2B). The enriched cells were induced to differentiate into mature brown adipocytes, and lipid accumulation was visualized by Oil-Red-O staining at 6 days after culturing with differentiation medium (Fig. 2C). Overexpression of wild-type PTPRB resulted in a lower degree of lipid accumulation compared with that of the control cells and those expressing the PTPRB mutant protein (Fig. 2C). Consistent with these results, the expression levels of brown adipocyte-related genes, such as UCP-1, PGC-1 $\alpha$, PRDM16, PPAR- $\gamma$ and CIDEA, were significantly down-regulated upon PTPRB overexpression but were not changed by the introduction of the inactive mutant PTPRB (Fig. 2D). These results suggest that the phosphatase activity is critical in the regulation of brown adipocyte differentiation. In particular, expression of key thermogenic genes UCP-1 and PGC-1 $\alpha$ was markedly suppressed by the ectopic PTPRB expression implying that PTPRB may induce a reduction of brown adipogenesis by decreasing the thermogenic program during differentiation.

Mitochondria have a central role in energy metabolism in many cell types. In particular, brown adipocytes contain a high number of mitochondria. Therefore, the mitochondrial content is an important indicator of brown adipocyte function and differentiation. To determine whether PTPRB influences the mitochondrial content of brown adipocytes, we examined the change in the expression level of mitochondrial NADH dehydrogenase 1 (ND1) during brown adipogenesis. As shown in Fig. 3A, the mRNA level of ND1 was significantly decreased by PTPRB overexpression; however, PTPRB-CS overexpression did not affect the ND1 level. Consistently, the results of the mitochondrial staining clearly show a decrease in mitochondria upon PTPRB overexpression (Fig. 3B). These results suggest that PTPRB affects mitochondrial biogenesis and the function of brown adipocytes by controlling the thermogenic gene expression of genes such as UCP-1 and PGC- $1 \alpha$.

PTPRB is a receptor-type PTP, which is known as VEPTP, because it is exclusively expressed in endothelial cells. PTPRB binds to vascular E-cadherin (VE-cadherin) and reduces the tyrosine phosphorylation of VE-cadherin independently of its enzymatic activity [28]. In addition, PTPRB regulates the VEGFR2 activity thereby modulating the VEGF-response [19]. VEGF is known to be a key factor in BAT development and maintenance [20]. Therefore, we speculated that PTPRB inhibited brown adipocyte differentiation by modulating VEGFR2 phosphorylation. Tyrosine phosphorylation of VEGFR2 in brown preadipocytes was rapidly increased in response to a differentiationinducing cocktail. Notably, overexpression of PTPRB led to the inhibition of tyrosine phosphorylation of VEGFR2 in pro-brown adipogenic culture conditions (Fig. 4A). However, the VEGFR2 phosphorylation level was not changed by the
A

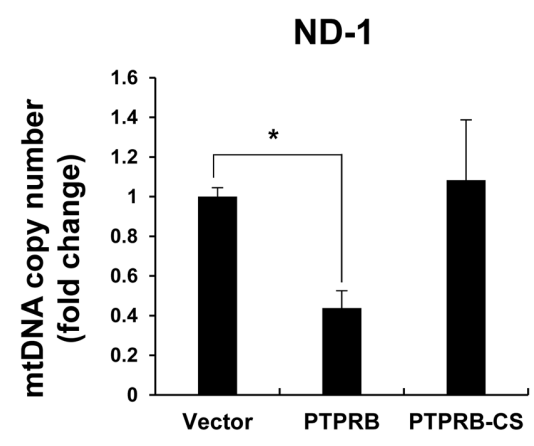

B

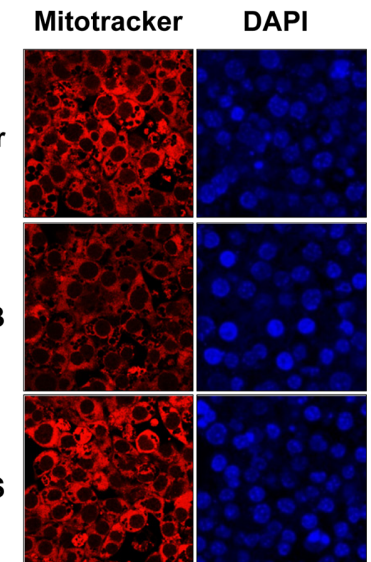

Merge

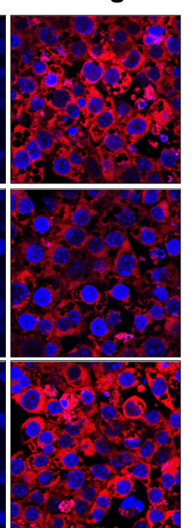

Fig. 3. Effect of PTPRB on the mitochondrial content of brown adipocytes.

(A) NADH dehydrogenase 1 (ND1) was used to measure the mitochondrial DNA (mtDNA). Genomic and mtDNA were isolated from brown adipocytes using DNA Mini Kits. TATA box binding protein (TBP) primers were used to assess the nuclear DNA. (B) The amount of mitochondria was assessed after ectopic expression of the wild-type PTPRB or PTPRB mutant. Cells were stained with MitoTracker fluorescence (Invitrogen) for visualization of mitochondria using confocal microscopy. MitoTracker is identified by the red color, and DAPI-stained nuclei are blue. 
A

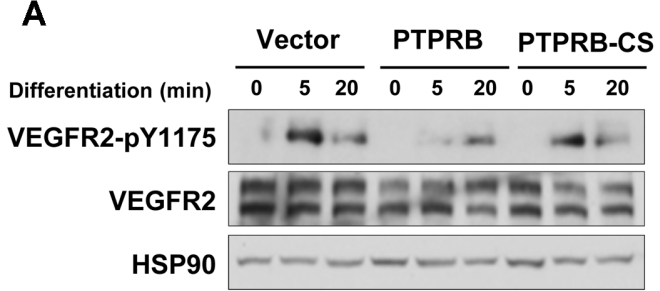

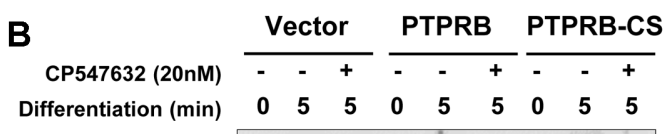

VEGFR2-pY1175

VEGFR2 $=m-m=-m$

HSP9O

C

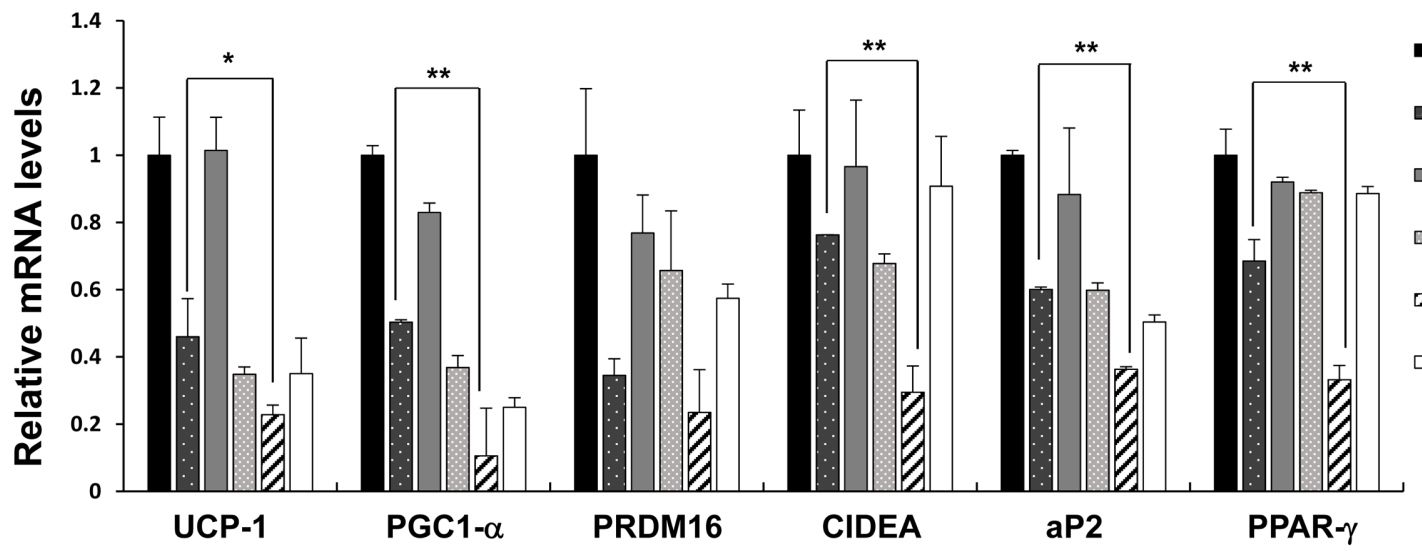

- Vector

๑PTPRB

口PTPRB-CS

$\square$ Vector + CP547632

DPTPRB + CP547632

口PTPRB-CS + CP547632

Fig. 4. PTPRB controls brown adipogenic differentiation by modulation of VEGFR2 phosphorylation.

(A) The changes in the phosphorylation level of VEGFR2 during brown adipogenesis were monitored by western blot analysis. Cells overexpressing wild-type PTPRB or catalytic inactive mutant PTPRB were serum-starved for $12 \mathrm{~h}$ and treated with a differentiation-inducing agent for the indicated times. (B) The phosphorylation level of VEGFR2 was confirmed during brown adipocyte differentiation in the absence or presence of CP547632 (pharmacological inhibitor of VEGFR2 phosphorylation). (C) The expression levels of brown adipogenic genes were analyzed by real-time PCR. PTPRB or PTPR-CS expressing brown preadipocytes in the absence or presence of CP547632 were induced to undergo brown adipogenic differentiation for 6 days according to standard procedures. Total RNA was extracted on day 6 of the differentiation. The data represent the means $\pm \mathrm{SD}\left(n=3,{ }^{*} p<0.05\right)$.

introduction of the catalytically inactive mutant PTPRB. To further clarify the importance of PTPRB in controlling VEGFR2 phosphorylation in brown adipogenesis, a specific inhibitor of VEGFR2 tyrosine phosphorylation, CP547632, was added to the culture medium during brown adipocyte differentiation. As shown in Fig. 4B, tyrosine phosphorylation of VEGFR2 was decreased with the treatment of CP547632. Moreover, the cells treated with CP547632 together with the PTPRB overexpression had an additive effect on the inhibition of VEGFR2 phosphorylation compared to cells treated only with CP547632. Additionally, comparison of CP547632-treated PTPRB overexpressed cells to the CP547632-only treated cell or the PTPRB overexpressed cell, showed that the expression of the brown adipogenic genes, such as UCP-1, PGC- $1 \alpha$., CIDEA and PPAR- $\gamma$, were significantly decreased. In general, VEGFR2 phosphorylation in brown preadipocytes was induced by serum VEGFresponse [20]. However, it is not clear whether the components of a differentiation-inducing cocktail, such as insulin, dexamethasone and IBMX, also activate VEGFR2 phosphorylation. Thus, extensive studies are needed to clarify the detailed mechanism of VEGFR2 phosphorylation during brown adipocyte differentiation.

In conclusion, we showed that PTPRB potently inhibits brown adipocyte differentiation through the regulation of VEGFR2 phosphorylation, which leads to a suppression of brown adipocyte-associated gene expression. Our data suggest that PTPRB might act as a novel modulator of brown adipogenesis, and it could be a novel target for the treatment of obesity.

\section{Acknowledgments}

This work was supported by grants from the KRIBB and from the Research Programs (grant nos. 2015M3A9D7029882, 2016R1C1B2013430, 2017M3A9C4065954, and 2017R1E1A 1A01074745) through the National Research Foundation of Korea. 


\section{Conflict of Interest}

The authors have no financial conflicts of interest to declare.

\section{References}

1. Bae K-H, Kim WK, Lee SC. 2012. Involvement of protein tyrosine phosphatases in adipogenesis: new anti-obesityk targets. BMB Rep. 45: 700-706.

2. Park A, Kim WK, Bae K-H. 2014. Distinction of white, beige and brown adipocytes derived from mesenchymal stem cells. World J. Stem Cells 6: 33-42.

3. Cypess AM, Lehman S, Williams G, Tal I, Rodman D, Goldfine $\mathrm{AB}$, et al. 2009. Identification and importance of brown adipose tissue in adult humans. N. Engl. J. Med. 360: 1509-1517.

4. Harms M, Seale P. 2013. Brown and beige fat: development, function and therapeutic potential. Nat. Med. 19: 1252-1263.

5. Townsend KL, Tseng YH. 2012. Brown adipose tissue: recent insights into development, metabolic function, and therapeutic potential. Adipocyte 1: 13-24.

6. Virtanen KA, Lidell ME, Orava J, Heglind M, Westergren R, Niemi T, et al. 2009. Functional brown adipose tissue in healthy adults. N. Engl. J. Med. 360: 1518-1525.

7. Hunter T. 1987. A thousand and one protein kinases. Cell 50: 823-829.

8. Mustelin GS, Feng N, Bottini A, Alonso A, Kholod N, Birle D, et al. 2002. Protein tyrosine phosphatases. Front Biosci. 7: 85-142.

9. Ostman A, Hellberg C, Bohmer FD. 2006. Protein tyrosine phosphatases and cancer. Nat. Rev. Cancer 6: 307-320.

10. Choi HR, Kim WK, Kim EY, Jung H, Kim JH, Han BS, et al. 2012. Protein tyrosine phosphatase profiling analysis of HIB-1B cells during brown adipogenesis. J. Microbiol. Biotechnol. 22: 1029-1033.

11. Choi HR, Kim WK, Kim EY, Han BS, Min JK, Chi SW, et al. 2013. Dual specificity phosphatase 10 controls brown adipocyte differentiation by modulating the phosphorylation of p38 mitogen-activated protein kinase. PLoS One 8: e72340.

12. Kim WK, Jung H, Kim D-H, Kim EY, Chung JW, Cho YS, et al. 2009. Regulation of adipogenic differentiation by LAR tyrosine phosphatase in human mesenchymal stem cells and 3T3-L1 preadipocytes. J. Cell Sci. 122: 4160-4167.

13. Kim WK, Bae K-H, Choi HR, Kim D-H, Choi KS, Cho YS, et al. 2010. Leukocyte common antigen-related (LAR) tyrosine phosphatase positively regulates osteoblast differentiation by modulating extracellular signal-regulated kinase (ERK) activation. Mol. Cells 30: 335-340.

14. Kim WK, Jung H, Kim EY, Kim D-H, Cho YS, Park BC, et al. 2011. RPTP $\mu$ tyrosine phosphatase promotes adipogenic differentiation via modulation of p120 catenin phosphorylation. Mol. Biol. Cell 22: 4883-4891.

15. Kim WK, Oh K-J, Choi HR, Park A, Han BS, Chi SW, et al. 2015. MAP kinase phosphatase 3 inhibits brown adipocyte differentiation via regulation of Erk phosphorylation, Mol. Cell. Endocrinol. 416: 70-76.

16. Choi HR, Kim WK, Park A, Jung H, Han BS, Lee SC, et al. 2013 Protein tyrosine phosphatase profiling studies during brown adipogenic differentiation of mouse primary brown preadipocytes. BMB Rep. 46: 539-543.

17. Bäumer S, Keller L, Holtmann A, Funke R, August B, Gamp A, et al. 2006. Vascular endothelial cell-specific phosphotyrosine phosphatase (VE-PTP) activity is required for blood vessel development. Blood 107: 4754-4762.

18. Winderlich M, Keller L, Cagna G, Broermann A, Kamenyeva O, Kiefer F, et al. 2009. VE-PTP controls blood vessel development by balancing Tie-2 activity. J. Cell Biol. 185: 657-671.

19. Hayashi M, Majumdar A, Li X, Adler J, Sun Z, Vertuani S, et al. 2013. VE-PTP regulates VEGFR2 activity in stalk cells to establish endothelial cell polarity and lumen formation. Nat. Commun. 4: 1672.

20. Bagchi M, Kim LA, Boucher J, Walshe TE, Kahn CR, D'Amore PA. 2013. Vascular endothelial growth factor is important for brown adipose tissue development and maintenance. FASEB J. 27: 3257-3271.

21. Son MJ, Kim WK, Kwak M, Oh K-J, Chang WS, Min J-K, et al. 2015. Silica nanoparticles inhibit brown adipocyte differentiation via regulation of $\mathrm{p} 38$ phosphorylation. Nanotechnology 26: 435101.

22. Son MJ, Kim WK, Park A, Oh K-J, Kim JH, Han BS, et al. 2016. Set7/9, a methyltransferase, regulates the thermogenic program during brown adipocyte differentiation through the modulation of p53 acetylation. Mol. Cell. Endocrinol. 431: 46-53.

23. Byun SK, An TH, Son MJ, Lee D, Kang HS, Lee EW, et al. 2017. HDAC11 inhibits myoblast differentiation through repression of MyoD-dependent transcription. Mol. Cells 40: 667-676.

24. Lee D, Choi H, Han BS, Kim WK, Lee SC, Oh K-J, et al. 2016. c-Jun regulates adipocyte differentiation via the KLF15mediated mode. Biochem. Biophys. Res. Commun. 469: 552-558.

25. Lee EW, Oh W, Song HP, Kim WK. 2017. Phosphorylation of p53 at threonine 155 is required for Jab1-mediated nuclear export of p53. BMB Rep. 50: 373-378.

26. Son MJ, Kim WK, Oh K-J, Park A, Lee D, Han BS, et al. 2016. Methyltransferase and demethylase profiling studies during brown adipocyte differentiation. BMB Rep. 49: 388-393.

27. Xue R, Lynes MD, Dreyfuss JM, Shamsi F, Schulz TJ, Zhang H, et al. 2015. Clonal analyses and gene profiling identify genetic biomarkers of the thermogenic potential of human brown and white preadipocytes. Nat. Med. 21: 760-768.

28. Nawroth R, Poell G, Ranft A. 2002. VE-PTP and VEcadherin ectodomains interact to facilitate regulation of phosphorylation and cell contacts. EMBO J. 21: 4885-4895. 